\title{
LE FUMAROLE DI VULCANO (EOLIE): UN LABORATORIO CHIMICO NATURALE
}

\author{
Nota del s.c. CARLO MARIA GRAMACCIOLI (*)
}

(Adunanza del 15 dicembre 2011)

SuNTO, - Gli studi dei minerali delle fumarole vulcaniche costituiscono ormai da secoli una fonte di osservazioni di grande interesse, non solo per la geologia e vulcanologia, ma anche per la chimica, come viene documentato da una lunga serie di importanti pubblicazioni. Mentre la località più "classica" di tali studi é costituita dal Vesuvio, tuttavia in Europa, in questi ultimi anni l'isola di Vulcano nelle Eolie ha fornito e sta tuttora fornendo nuovi esempi di minerali delle fumarole, che offrono spunti per interessanti riflessioni.

ABSTRACT. - The minerals from volcanic fumaroles have long attracted the interest of scientists: among these persons, we find not only geologists or volcanologists in the strict sense, but chemists and physicists as well, with a long series of publications spanning an interval of more than three centuries. Whereas Vesuvius was the most "classical" locality for such observations until the last half of the 20th century, in recent times Vulcano in the Aeolian islands has become instead the most promising locality in Europe for such studies, which provide a series of important observations involving several disciplines.

Già dal Rinascimento, ma soprattutto dal primo sviluppo dell'età moderna, la particolare attenzione delle persone colte era stata rivolta ai vulcani, i più famosi dei quali si trovavano in Italia e spesso facevano parte del classico "grand tour", un itinerario culturale che $\mathrm{i}$

(*) Università degli Studi di Milano. 
primi "turisti" del Nord effettuavano. Tra questi cultori, troviamo non raramente personaggi d'eccezione, con un vastissimo campo di attività diverse, come GOETHE; tuttavia l'interesse per i vulcani (in particolare il Vesuvio) non era ristretto soltanto agli scrittori ed artisti in genere, oppure ai geologi ed ai naturalisti, tra cui basterà citare il celebre DoLOMIEU, che come sappiamo visitò non solo il Vesuvio, ma anche le Eolie, o SPALLANZANI, ma era attivamente sentito e diffuso anche tra $\mathrm{i}$ cultori di altre scienze, tra cui la fisica ed in particolare la chimica, assieme alla cristallografia ed alla mineralogia. Tra i famosi chimici del Sette-Ottocento che si occuparono attivamente di vulcani, abbiamo infatti Lémery, Thomson, Davy, Sainte-Claire Deville, CROOKES; si hanno poi i cristallografi, quali De LisLe, HaÜY, VOM RATH, ecc. ecc., per non parlare poi di una serie di illustri mineralogisti, tra cui Fougeroux De Boundaroy, Breislak, Monticelli, COVELLI, SCACCHI, STRÜVER, e così via, per arrivare infine ai nomi del secolo ventesimo, tra i quali primo fra tutti fu ZAMBONINI. Non a caso si diceva già allora che i vulcani, tra cui soprattutto il Vesuvio, costituivano dei veri e propri laboratori chimici naturali, e questa tradizione continua ancora oggi.

Infatti, le scoperte dei minerali vulcanici, ed in particolare quelli delle fumarole, ben lungi dal rivestire un puro interesse naturalistico, se non addirittura soltanto collezionistico, anche oggi sono molto importanti per favorire il progresso della ricerca scientifica, ed interessano diverse discipline:

1) Geologia e vulcanologia: I minerali delle fumarole testimoniano la natura dei fluidi gassosi, di origine più o meno profonda, da cui derivano: si osservano infatti non solo lo zolfo, ma anche fluoruri, cloruri, acido borico, sali ammoniacali, ecc. che corrispondono alla presenza nei gas di notevoli quantità di composti solforati, di acidi alogenidrici, di vari alogenuri e di ammoniaca. La stessa presenza di quest'ultima sostanza si lega, come vedremo, ad ipotesi suggestive sulla sua origine dai magmi od in genere anche dalle zone più profonde della crosta o dello stesso mantello, e gli alogenuri, anche quelli insoliti in natura, illustrano seducenti possibilità di deposizione dei metalli da cloruri e fluoruri volatili, che possono applicarsi alle teorie sull'origine dei giacimenti; qui ed in altri celebri vulcani (vedi quelli della Kamchatka e delle isole Kurili dove compaiono addirittura minerali di renio, uno dei metalli più rari), spesso si verifica un sorprendente arricchimento 
di elementi molto scarsi in natura, quali ad esempio gli alogeni pesanti come bromo e iodio, oppure il tallio o lo stesso bismuto, che compaiono appunto con relativa frequenza nei prodotti depositati alla bocca dei crateri.

2) Chimica: Come molte delle vicende geologiche legate alle rocce più profonde, i vulcani in particolare ci rammentano che la chimica non riguarda solo le condizioni sperimentali più accessibili nei laboratori, ma anche altre più per così dire remote, ma certamente non meno interessanti ed utili. La termodinamica diventa fondamentale per studiare adeguatamente i complessi equilibri che si verificano nei fluidi gassosi, o tra questi e le fasi solide: qui ogni minerale, anche il più raro, è testimone di particolari condizioni chimico-fisiche (temperatura, pressione, concentrazione - più propriamente attività - dei diversi componenti, tra cui specie chimiche ancora poco note); concetti ancora più avanzati e delicati riguardano le possibili condizioni di non-equilibrio. Inoltre, si deve tenere conto della particolare complessità dei solfuri e di alcuni alogenuri, che li rendono quanto mai interessanti per la stessa chimica delle fasi cristalline e degli elementi di transizione.

3) Cristallografia: In molti dei minerali delle fumarole si sono riscontrate strutture di tipo nuovo e talora assai complesso, che ampliano la conoscenza dei composti inorganici (si vedano soprattutto $i$ solfuri e gli alogenuri complessi).

Mentre le più celebri fumarole vesuviane sono ormai praticamente inattive da più di sessant'anni, in Italia si hanno altri celebri esempi di questo tipo di formazione di minerali vulcanici. Attualmente il luogo più interessante é l'isola di Vulcano, nelle Eolie: questa isola, infatti, è la "località-tipo" per 25 specie mineralogiche di cui ben 17 scoperte nell' ambito delle nostre ricerche, ed è documentata la presenza nelle fumarole dell'isola di oltre 100 specie. Altre 30 fasi potenzialmente nuove sono state individuate, ma purtroppo i dati sono ancora insufficienti per farle approvare come nuove specie dalla Commissione internazionale (CNMNC): questo tuttavia non toglie il fatto che anche qui si abbia a che fare con altri casi molto interessanti ed unici al mondo. Una esauriente descrizione di tutti questi minerali e dell'ambiente in cui si sono formati viene fornita dal libro: "Vulcano - Tre secoli di mineralogia" di CAMPOSTRINI et al. (2011). (Tab. 1). 
Tab. 1 - Le nuove specie mineralogiche scoperte nelle fumarole di Vulcano.

\begin{tabular}{|c|c|}
\hline ADRANOSITE & $\left(\mathrm{NH}_{4}\right)_{4} \mathrm{NaAl}_{2}\left(\mathrm{SO}_{4}\right)_{4} \mathrm{Cl}(\mathrm{OH})$ \\
\hline AIOLOSITE & $\mathrm{Na}_{2}(\mathrm{Na}, \mathrm{Bi})\left(\mathrm{SO}_{4}\right)_{3} \mathrm{Cl}$ \\
\hline ALUMINOCOQUIMBITE & $\mathrm{AlFe}^{3+}\left(\mathrm{SO}_{4}\right)_{3} \cdot 9 \mathrm{H}_{2} \mathrm{O}$ \\
\hline ARGESITE & $\left(\mathrm{NH}_{4}\right)_{7} \mathrm{Bi}_{3} \mathrm{Cl}_{16}$ \\
\hline BARBERIITE & $\mathrm{NH}_{4} \mathrm{BF}_{4}$ \\
\hline BRONTESITE & $\left(\mathrm{NH}_{4}\right)_{3} \mathrm{PbCl}_{5}$ \\
\hline CANNIZZARITE & $\mathrm{Pb}_{46} \mathrm{Bi}_{54} \mathrm{~S}_{127}$ o $\mathrm{Pb}_{4} \mathrm{Bi}_{5-\mathrm{x}}(\mathrm{S}, \mathrm{Se})_{11,5-1,5 \mathrm{x}}$ \\
\hline COSSAITE & $\mathrm{Mg}_{0,5} \mathrm{Al}_{6}\left(\mathrm{SO}_{4}\right)_{6}\left(\mathrm{HSO}_{4}\right) \mathrm{F}_{6} 36 \mathrm{H}_{2} \mathrm{O}$ \\
\hline CLINOMETABORITE & $\mathrm{HBO}_{2}$ \\
\hline DEMARTINITE & $\mathrm{K}_{2} \mathrm{SiF}_{6}$ \\
\hline DEMICHELEITE-(Br) & $\mathrm{BiSBr}$ \\
\hline DEMICHELEITE-(Cl) & $\mathrm{BiSCl}$ \\
\hline DEMICHELEITE-(I) & BiSI \\
\hline HEPHAISTOSITE & $\mathrm{TlPb}_{2} \mathrm{Cl}_{5}$ \\
\hline HIERATITE & $\mathrm{K}_{2} \mathrm{SiF}_{6}$ \\
\hline KNASIBFITE & $\mathrm{K}_{3} \mathrm{Na}_{4}\left[\mathrm{SiF}_{6}\right]_{3}\left[\mathrm{BF}_{4}\right]$ \\
\hline LAFOSSAITE & $\mathrm{TlCl}$ \\
\hline MAGNESIOAUBERTITE & $(\mathrm{Cu}, \mathrm{Mg}) \mathrm{Al}\left(\mathrm{SO}_{4}\right)_{2} \mathrm{Cl} \cdot 14 \mathrm{H}_{2} \mathrm{O}$ \\
\hline MILLOSEVICHITE & $\left(\mathrm{Al}, \mathrm{Fe}^{3+}\right)_{2}\left(\mathrm{SO}_{4}\right)_{3}$ ?? \\
\hline MOZGOVAITE & $\mathrm{PbBi}_{4}(\mathrm{~S}, \mathrm{Se})_{3}$ \\
\hline PANICHIITE & $\left(\mathrm{NH}_{4}\right)_{2} \mathrm{SnCl}_{6}$ \\
\hline PYRACMONITE & $\left(\mathrm{NH}_{4}\right)_{3} \mathrm{Fe}^{3+}\left(\mathrm{SO}_{4}\right)_{3}$ \\
\hline STEROPESITE & $\mathrm{Tl}_{3} \mathrm{PbCl}_{6}$ \\
\hline THERMESSAITE & $\mathrm{K}_{2}\left[\mathrm{AlF}_{3} \mid \mathrm{SO}_{4}\right]$ \\
\hline VURROITE & $\mathrm{Pb}_{20} \mathrm{Sn}_{2}(\mathrm{Bi}, \mathrm{As})_{22} \mathrm{~S}_{54} \mathrm{Cl}_{6}$ \\
\hline
\end{tabular}




\section{CRISTALLOCHIMICA DEI PRODOTTI DELLE FUMAROLE}

\section{Solfuri e solfosali}

Particolarmente importanti dal punto di vista scientifico sono i solfuri, dei quali molti termini con arsenico, antimonio e bismuto vengono considerati da alcuni (ma non da tutti gli autori) come "solfosali", seguendo una tradizione che risale a BERZELIUS. La struttura dei solfuri e soprattutto quella dei solfosali si presenta in parecchi casi notevolmente complessa e praticamente unica nel suo genere, e per questo anche $\mathrm{i}$ composti naturali forniscono esempi quanto mai interessanti nella trattazione moderna delle teorie dei solidi.

Mentre quasi tutti gli altri minerali (ossidi, alogenuri e sali ossigenati) presentano un carattere prevalentemente ionico, e cioè gli atomi manifestano forti cariche elettriche da cui derivano intensi campi elettrostatici che ne determinano i legami chimici, nei solfuri (come negli arseniuri, seleniuri, tellururi, ecc. che vengono definiti soprattutto dai fisici come "calcogenuri") i legami sono invece di tipo essenzialmente covalente, con elettroni almeno in parte delocalizzati ed estesi a tutto il cristallo e non "relegati" all'interno delle varie molecole che qui addirittura quasi sempre non esistono (come del resto anche nei cristalli ionici). Nei calcogenuri spesso si manifestano vere e proprie onde di densità elettronica, la cui periodicità non ha in generale nulla a che fare con quella dei reticoli cristallini e si verifica una corrispondenza con quanto si osserva nelle trattazioni quantomeccaniche degli orbitali molecolari e delle vibrazioni dei reticoli (fondamentali a questo proposito sono le zone di Brillouin); sulla base di tutto questo non è sorprendente l'esistenza appunto dei reticoli incommensurabili, dove cioè ripetizione cristallografica e periodicità composizionale possono diventare addirittura incompatibili. In questi minerali, spesso a carattere "semimetallico" (molti di essi presentano importanti proprietà di semiconduttori) possono diventare importanti gli interstiziali, oppure le "vacanze", cioè dei vuoti strutturali che, insieme ad altri "difetti" della struttura ordinata, possono arrivare a rendere le formule chimiche addirittura "non stechiometriche" e cioè non esprimibili con coefficienti interi, violando le comuni regole "classiche" della chimica con cui non raramente, ci si sforza di interpretare la composizione usando formule assai complesse. Questi composti possono inoltre manifestare politipismo, e cioè possono dare origine a fasi solide molto simili tra loro, che differi- 
scono soltanto nel tipo e nella periodicità di impacchettamento degli strati, che per i veri politipi sono tutti uguali (o quasi) nella composizione, ma si hanno anche variazioni; molto utili, se non addirittura essenziali, a questo riguardo, sono gli studi a microscopia elettronica (TEM) ad alta risoluzione.

Tutte queste particolarità strutturali, che rendono le strutture assai complesse e che divergono notevolmente dai comuni assunti della chimica "classica", si manifestano spesso con particolare evidenza nei solfuri del piombo con altri metalli, tra i quali soprattutto appunto il bismuto, lo stagno, l'arsenico e l' antimonio: inutile dire che il loro studio approfondito contribuisca notevolmente ad allargare gli orizzonti della conoscenza scientifica e dell'insegnamento di una chimica veramente moderna collegandola alle recenti teorie dello stato solido.

Nelle fumarole della nostra isola, molto interessanti sono i solfuri di bismuto, e soprattutto quelli che contengono anche piombo, alcuni dei quali sono molto simili a quanto si trova in alcune celebri miniere della Svezia (anche specie selenifere). Le miniere della Svezia non sono giaciture di tipo vulcanico recente, per cui quanto si osserva a Vulcano può essere un esempio di formazione di parecchi giacimenti "classici" metalliferi, che qui si può osservare direttamente. Non in tutti i vulcani compaiono minerali di bismuto (ad es. al Vesuvio questi sono assenti): qui invece i solfuri di bismuto, spesso con piombo ed altri metalli, risultano particolarmente abbondanti, al punto di fare considerare la nostra isola come una località "classica" per queste specie, che si manifestano soprattutto nei periodi di maggiore attività. Tra questi solfuri, oltre alla bismutinite $\mathrm{Bi}_{2} \mathrm{~S}_{3}$, un minerale qui addirittura comune, ed alla cannizzarite $\mathrm{Pb}_{4} \mathrm{Bi}_{5-\mathrm{x}}(\mathrm{S}, \mathrm{Se})_{11,5-1,5 \mathrm{x}}$, che era già stata qui scoperta per la prima volta nel mondo (1924) da ZAMBONINI, DE FIORE e CAROBBI; molto più recentemente (PINTO et al., 2006ab, GARAVELLI et al., 2005) sono state riconosciute anche la galena $\mathrm{PbS}$, la galenobismutite $\mathrm{PbBi}_{2} \mathrm{~S}_{4}$, la lillianite $\mathrm{Pb}_{3} \mathrm{Bi}_{2} \mathrm{~S}_{6}$ (non argentifera), la kirkiite $\mathrm{Pb}_{10} \mathrm{Bi}_{3} \mathrm{As}_{3} \mathrm{~S}_{19}$, la wittite $\mathrm{Pb}_{3} \mathrm{Bi}_{4}(\mathrm{~S}, \mathrm{Se})_{9}$, l'heyrovskýite $\mathrm{Pb}_{6} \mathrm{Bi}_{2} \mathrm{~S}_{9}$ (cadmifera), la mozgovaite $\mathrm{PbBi}_{4}(\mathrm{~S}, \mathrm{Se})_{7}$ e la vurroite $\mathrm{Pb}_{20} \mathrm{Sn}_{2}(\mathrm{Bi}, \mathrm{As})_{22} \mathrm{~S}_{54} \mathrm{Cl}_{6}$.

Relativamente frequente è la sostituzione dello zolfo con gli alogeni (soprattutto, ma non esclusivamente, cloro), che porta, come caso limite, a dei veri e propri solfoalogenuri, praticamente unici in natura, ma ben noti nel campo dei componenti usati nell'industria elettronica: questi minerali (DEMARTIN et al., 2008b, 2009d, 2010c), ed altri prodotti osservati sinora soltanto come depositi entro tubi di quarzo diret- 
tamente introdotti nelle fumarole (vedi GARAVELLI et al. 1997, 2005; VURRO et al. 1999, e riferimenti ivi citati), suggeriscono che la deposizione di questi minerali é avvenuta per reazione del tricloruro di bismuto, che é volatile, con idrogeno solforato od altri gas solforati presenti nei vapori.

Singolare è la virtuale assenza a Vulcano di solfuri non solo di antimonio, ma anche di arsenico, come il realgar $\left(\mathrm{As}_{4} \mathrm{~S}_{4}\right)$ che è invece largamente diffuso in altri vulcani: qui si hanno, seppure molto raramente, solo due solfosali, la kirkiite $\mathrm{Pb}_{10} \mathrm{Bi}_{3} \mathrm{As}_{3} \mathrm{~S}_{19}$ e la vurroite $\mathrm{Pb}_{20} \mathrm{Sn}_{2}(\mathrm{Bi}, \mathrm{As})_{22} \mathrm{~S}_{54} \mathrm{Cl}_{6}$ che contengono quantità significative di questo elemento. E possibile che questa assenza nei minerali sia dovuta soprattutto all'alta temperatura delle fumarole della Fossa ed alla presenza di notevoli concentrazioni di cloruri, che rendono l'arsenico molto volatile, sotto forma del tricloruro $\mathrm{AsCl}_{3}$, impedendone la deposizione nei prodotti solidi.

Una caratteristica interessante dei solfuri piombiferi e bismutiferi di Vulcano (galenobismutite, lillianite) è quella di contenere a volte quantità non trascurabili di cloro, che diventano addirittura essenziali nella vurroite (GARAVELLI et al., 2005; PINTO et al., 2006a). Questi dati confermano quanto si era già osservato in questi ultimi anni in alcuni minerali e persino in campioni sintetici quali $\mathrm{Bi}_{4} \mathrm{Cl}_{2} \mathrm{~S}_{5}$, dove si osserva una sostituzione statisticamente disordinata dello zolfo con il cloro (KRÄMER, 1979; GARAVELLI et al., 1997). Un ragionamento simile all'esempio del cloro vale anche per altri alogeni, come il bromo, per cui ad esempio, la vurroite è anche sensibilmente bromifera; seguendo questa linea, non desta sorpresa la presenza nelle fumarole vulcaniche persino di termini iodiferi, come è stato effettivamente riscontrato soprattutto al vulcano Mutnovski in Kamchatka: in questa località lo iodio prevale addirittura sugli altri alogeni nel raro solfuro mutnovskite $\mathrm{Pb}_{2} \mathrm{AsS}_{3}(\mathrm{I}, \mathrm{Cl}, \mathrm{Br}$ ) (ZELENSKI et al., 2006). A Vulcano questa specie non è stata ancora osservata; esistono comunque quantità significative di iodio anche in alcuni minerali già noti di questa località.

Nella galenobismutite $\mathrm{PbBi}_{2} \mathrm{~S}_{4}$ di Vulcano è stato dimostrato un meccanismo interessante di sostituzione dello zolfo con il cloro, che avviene contemporaneamente a quella del piombo con il bismuto secondo lo schema: $\mathrm{Pb}+\mathrm{Cl} \leftrightarrow \mathrm{Bi}+\mathrm{S}$ (PINTO et al. 2006a). Questo stesso schema, che ricorda alquanto il motivo strutturale delle demicheleiti, potrebbe manifestarsi anche negli altri solfuri, non solo, ma persino in alcuni alogenuri (cotunnite $\mathrm{PbCl}_{2}$, challacolloite $\mathrm{KPb}_{2} \mathrm{Cl}_{5}$ ), spiegan- 
do i toni di colore che vanno dal giallo sino al rosso ed al bruno scuro che si osservano proprio a Vulcano, in stretta associazione con minerali di bismuto mentre altrove, questi cloruri sono invece sempre incolori o bianchi e il bismuto è assente, almeno in quantità rilevanti. Mancano purtroppo ancora oggi studi accurati che confermino o contraddicano chiaramente queste supposizioni.

\section{Il selenio}

Un altro punto importante per tutti i solfuri bismutiferi di Vulcano è la presenza di quantità non trascurabili di selenio che sostituisce facilmente lo zolfo, un fenomeno questo che si verifica non di rado non soltanto per gli esemplari delle fumarole di altri vulcani (ad es. quelli della Kamchatka) ma anche per altri campioni "classici" di queste stesse specie o di specie vicine, quali la wittite, ecc. quali si trovano soprattutto in celebri miniere della Svezia (Gladhammar, Falun) e che non hanno origine vulcanica recente. Considerando anche questi particolari, e tenendo conto anche della presenza di oro e tellurio come inclusioni microscopiche (Fulignati \& SBRANA, 1998), non si può fare a meno di continuare a pensare che la deposizione di questi solfuri metallici alla superficie dei vulcani non sia in sostanza troppo diversa da ciò che è avvenuto anche all'interno della Terra: se questo è verosimile, qui noi oggi abbiamo la possibilità di osservare un vero e proprio giacimento metallifero che si sta formando, avendo così la possibilità di verificare direttamente anche il tipo dei fluidi mineralizzanti, che almeno in parecchi casi potrebbero non essere troppo diversi da quelli delle fumarole.

Come per lo zolfo che a Vulcano è abbondante allo stato nativo, è stato osservato qui recentemente anche il selenio nativo, già noto da lungo tempo, ma questa volta in campioni di natura incontrovertibile.

L'arricchimento del selenio che sostituisce lo zolfo (due elementi questi che sono "omologhi" e cioè che stanno immediatamente uno sotto l'altro nella celebre tabella di Mendelejev, ed i cui atomi hanno struttura elettronica e proprietà chimiche assai simili) probabilmente dipende da un fenomeno simile a quello che succede per gli alogeni (bromo ed anche iodio, che sostituiscono il cloro talora in maniera considerevole), ed è legato alle notevoli differenze di massa degli atomi, che si riflette piuttosto nelle proprietà fisiche (volatilità) che nelle proprietà chimiche. Queste ultime tuttavia a volte diventano dominanti: ci si può chiedere allora, ad esempio, come mai i numerosi solfati che si incontrano in gran copia nelle 
fumarole non siano così seleniferi come lo sono invece i solfuri: qui interviene tuttavia una notevole differenza che si incontra tra l'ossidabilità dello zolfo, che passa molto facilmente allo stato di ossidazione più alto (6+ nei solfati) rispetto all'omologo selenio, che pur dando luogo ai seleniati (isostrutturali e spesso isomorfi con i solfati), è molto più difficilmente ossidabile e tende a rimanere al massimo allo stato 4+ come nei seleniti; un caso parallelo si riscontra anche tra gli omologhi cloro e bromo, ove lo stato $7+$, presente nei perbromati, è stato individuato in laboratorio solo non molti anni fa, mentre i perclorati sono prodotti chimici noti da lungo tempo. In effetti, i seleniti, che costituiscono la quasi totalità dei sali ossigenati del selenio che si osservano in natura, sono vicini strutturalmente ai solfiti, che sono invece facilmente ossidabili ed assai rari nei minerali. Quindi, mentre in natura selenio e zolfo si sostituiscono facilmente a vicenda nei solfuri, nelle specie ossigenate questo invece non succede, se non eccezionalmente.

\section{Altri minerali di bismuto}

Abbiamo già visto l'importanza dei solfuri di bismuto a Vulcano; recentemente sono stati trovati anche alogenuri veri e propri, come è il caso soprattutto della steropesite $\mathrm{Tl}_{3} \mathrm{BiCl}_{6}$ (DEMARTIN et al., 2009b) che accompagna la lafossaite $\mathrm{TlCl}$, ed anche dell'argesite $\left(\mathrm{NH}_{4}\right)_{7} \mathrm{Bi}_{3} \mathrm{Cl}_{16}$ (DEMARTIN et al., 2012), oltre a diverse altre specie non ancora studiate; si ha anche - non troppo raramente - l'ossicloruro bismoclite $\mathrm{BiOCl}$ in eccezionali campioni ben cristallizzati, un caso questo unico al mondo (CAMPOSTRINI et al., 2011). La presenza degli alogenuri di bismuto, alcuni dei quali sono stati riscontrati anche da GARAVELLI et al. (1997) nei tubi di quarzo immersi in queste fumarole, è un altro indizio del ruolo importante che rivestono i cloruri (e gli alogenuri in genere) nel trasportare sotto forma di composti volatili i metalli pesanti, e che può risultare particolarmente interessante nello studio delle origini dei giacimenti metalliferi.

Non è affatto escluso che parecchi degli alogenuri e dei solfoalogenuri osservati sinora soltanto come depositati in questi tubi di quarzo in diversi vulcani (soprattutto qui ed in Kamchatka), e pertanto non considerati come minerali validi dalla Commissione dell'IMA preposta alla loro approvazione in conformità alle regole internazionali, non si possano trovare anche direttamente sul terreno, come è già successo per molte delle nuove specie di Vulcano, e quindi risulta più che probabile la futura scoperta di un numero rilevante di altri nuovi minerali 
di composizione molto interessante (alogenuri di cadmio, cloruri di piombo e potassio del tipo di $\mathrm{KPbCl}_{3}$, solfocloruri di arsenico, bromuri e ioduri di tallio, cadmio, ecc.ecc.).

Oltre a veri e propri cloruri di bismuto, nelle fumarole si è scoperta anche la aiolosite $\mathrm{Na}{ }_{4} \mathrm{Bi}\left(\mathrm{SO}_{4}\right)_{3} \mathrm{Cl}$ (DEMARTiN et al., 2010d). Questo nuovo minerale, essenzialmente un solfato, ma che pure contiene cloro in quantità subordinata, ha la struttura dell'apatite $\mathrm{Ca} 5\left(\mathrm{PO}_{4}\right)_{3} \mathrm{~F}$, o meglio, dell'hedyphane $\mathrm{Pb}_{3} \mathrm{Ca}_{2}\left(\mathrm{AsO}_{4}\right)_{3} \mathrm{Cl}$, e si è verosimilmente formato per azione degli acidi delle fumarole $\left(\mathrm{H}_{2} \mathrm{SO}_{4}, \mathrm{HCl}\right)$ sulla bismutinite già depositata.

\section{Alogenuri}

Nelle fumarole sono presenti parecchi cloruri, tra cui soprattutto quello d'ammonio. Molto più rari sono invece quelli di metalli pesanti, tra i quali soprattutto il piombo, il ferro, il bismuto, che formano di fatto interessanti complessi, per alcuni dei quali la struttura cristallina è stata risolta per la prima volta. Tra questi si ha ad esempio l'argesite, $\left(\mathrm{NH}_{4}\right)_{7} \mathrm{Bi}_{3} \mathrm{Cl}_{16}$, che é il primo esempio noto dell'anione $\left[\mathrm{Bi}_{2} \mathrm{Cl}_{10}\right]^{4}$ (DEMARTIN et al., 2012).

Assieme ai solfuri, importanti e spesso unici sono gli alogenuri, e cioè i composti che derivano dall'unione di vari metalli con il cloro, il fluoro, ed anche con il bromo o lo iodio, questi ultimi assai più rari dei precedenti. Intermedi tra gli alogenuri ed i solfuri sono specie singolari, come la demicheleite-(Br) BiSBr (DEMARTIN et al., 2008b) od anche i suoi equivalenti dove prevalgono il cloro e lo iodio sul bromo, come la demicheleite- $(\mathrm{Cl}) \mathrm{BiSCl}$, e persino il solfoioduro BiSI. Quest'ultimo, riscontrato in natura solo molto recentemente (DEMARTIN et al., 2010c) era stato già osservato entro tubi di quarzo introdotti nelle fumarole del Mutnovski, in Kamchatka (ZELENSKI e BORTNIKOVA, 2005); un altro solfoalogenuro $\mathrm{Bi}_{19} \mathrm{Cl}_{3} \mathrm{~S}_{27}$ era stato già notato da GARAVELLI et al. (1997) nei tubi di quarzo introdotti nelle fumarole di Vulcano, enfatizzando il ruolo dei cloruri nel trasporto del bismuto nei gas vulcanici.

Le demicheleiti, oltre a confermare questa possibilità del trasporto di $\mathrm{Bi}$, ci forniscono un rarissimo esempio naturale dove il bromo può prevalere sul cloro, evidenziandone l'arricchimento nei prodotti delle fumarole, un fatto questo già notato da molto tempo (in tono molto minore) anche per altri minerali, come il clorammonio $\mathrm{NH}_{4} \mathrm{Cl}$, che è spesso sensibilmente bromifero (CORADOSSI et al., 1985, 1996), nonché 
più recentemente per la vurroite $\mathrm{Pb}_{20} \mathrm{Sn}_{2}(\mathrm{Bi}, \mathrm{As})_{22} \mathrm{~S}_{54} \mathrm{Cl}_{6}$ (GARAVELLI et al. 2005). Un arricchimento in bromo e iodio, in maniera questa volta notevole, si verifica anche per gli alogenuri di tallio, come la lafossaite $\mathrm{Tl}\left(\mathrm{Cl}, \mathrm{Br}\right.$ ) (ROBERTS et al. 2006), l'hephaistosite $\mathrm{TlPb}_{2} \mathrm{Cl}_{5}$ (CAMPOSTRINI et al., 2008), la steropesite $\mathrm{Tl}_{3} \mathrm{BiCl}_{6}$ (DEMARTIN et al., 2009). In alcuni casi, anche qui gli alogeni più pesanti possono addirittura prevalere, e si arriva al bromuro TlBr e persino allo ioduro TlI quasi puri. Tuttavia i campioni di queste sostanze finora trovati a Vulcano (CAMPOSTRINI et al., 2011) sono troppo esigui e male cristallizzati per fornire dati indiscutibili da sottoporre alla commissione IMA per l'approvazione delle rispettive specie, anche se l'analisi chimica non lascia dubbi.

L'arricchimento del bromo (e dello iodio) in questi minerali delle fumarole è probabilmente dovuto a diversi fattori legati alla differenza di massa degli atomi che si riflette nelle proprietà termodinamiche, e cioè, ad esempio: 1) esiste già un rapporto tra $\mathrm{Br}$ e $\mathrm{Cl}$ maggiore del solito nei gas stessi, che deriva probabilmente da un'origine almeno parziale di questi gas legata ad un magma profondo; 2) da un frazionamento del bromo che si verifica all'atto stesso della deposizione dei prodotti solidi all'orlo delle fumarole, dato che i bromuri sono in generale meno volatili dei cloruri.

Le fumarole del cratere della Fossa hanno fornito esempi di fluoruri naturali, tra cui quasi esclusivamente sali di acidi complessi, quali esafluorosilicati (fluosilicati), tetrafluoroborati (fluoborati) e fluoalluminati di vario tipo, spesso nuovi.

Vista la non volatilità dei fluoruri di alluminio, si presume che i fluoalluminati delle fumarole siano il prodotto dell'attacco delle rocce alluminifere da parte degli acidi fluoridrico e solforico, mentre i fluosilicati (almeno in parte) e soprattutto i fluoborati derivino dalla deposizione da gas quali $\mathrm{SiF}_{4}$ e $\mathrm{BF}_{3}$.

Il primo fluoruro scoperto a Vulcano, la hieratite $\mathrm{K}_{2} \mathrm{SiF}_{6}$, fu osservato qui per la prima volta nel mondo già alla fine dell'Ottocento (Cossa 1881-82,1882,1884), precedendo addirittura la scoperta di questa stessa specie al Vesuvio (ZAMBONINI \& CAROBBI, 1926). Più che composti "semplici" quali si hanno assai più comunemente nelle altre parti del mondo (p.es. la fluorite $\mathrm{CaF}_{2}$, la villiaumite $\mathrm{NaF}$, ecc.), quasi tutti i fluoruri delle fumarole vulcaniche possono venire piuttosto considerati invece come sali di acidi complessi fluorurati contenenti elementi quali il silicio od il boro (acido esafluorosilicico $\mathrm{H}_{2} \mathrm{SiF}_{6}$, acido tetrafluoroborico $\mathrm{HBF}_{4}$ ), e talora anche l'alluminio. Tra queste specie a 
Vulcano si hanno - oltre alla rara hieratite- anche la dimorfa demartinite, pure $\mathrm{K}_{2} \mathrm{SiF}_{6}$, scoperta non troppi anni fa in bei cristallini esagonali piramidali (GRAMACCIOLI \& CAMPOSTRINI, 2007) accompagnata dall'avogadrite $\mathrm{KBF}_{4}$, un minerale questo isostrutturale con la barite $\mathrm{BaSO}_{4}$ e che fu per la prima volta osservata al Vesuvio (ZAMBONINI, 1926), e la barberiite $\mathrm{NH}_{4} \mathrm{BF}_{4}$, scoperta da GARAVELLI e VURRO nel 1994. Come recentissima novità, qui è stato trovato per la prima volta nel mondo uno strano minerale, la knasibfite $\mathrm{K}_{3} \mathrm{Na}_{4}\left[\mathrm{SiF}_{6}\right]_{3}\left[\mathrm{BF}_{4}\right]$ (DEMARTIN et al. 2008a), che contiene entrambi gli anioni esafluorosilicato e tetrafluoroborato, un esempio che finora non era mai stato osservato nemmeno nei prodotti sintetici. Oltre alla malladrite $\mathrm{Na}_{2} \mathrm{SiF}_{6}$, pure trovata recentemente (GARAVELLI \& VURRO, 1994, GARAVELLI et al., 1997), ci si aspetterebbe di trovare a Vulcano anche altri esafluorosilicati o tetrafluoroborati tipici delle fumarole vesuviane, tra cui particolarmente la cryptohalite $\left(\mathrm{NH}_{4}\right)_{2} \mathrm{SiF}_{6}$, ma nonostante ricerche accurate questo minerale non é stato trovato finora nella nostra località, per quanto possa essere facilmente sfuggito ai ricercatori per il suo aspetto assolutamente incospicuo (da cui lo stesso nome della specie).

Di un gruppo assai caratteristico di fluoruri complessi con alluminio, che soprattutto in Groenlandia (Ivigtut) ha fornito in abbondanza numerosi esempi, a Vulcano è stata osservata parecchi anni fa (BERNAUER, 1941) solamente la gearksutite $\mathrm{CaAl}(\mathrm{OH}) \mathrm{F}_{4} \cdot \mathrm{H}_{2} \mathrm{O}$; si ha qui invece, non troppo raramente, un minerale caratteristico, la thermessaite $\mathrm{K}_{2}\left[\mathrm{AlF}_{3} \mid \mathrm{SO}_{4}\right]$ (DEMARTIN et al., 2008b). Questa contiene anche solfati, e la struttura presenta catene di ottaedri $\mathrm{AlF}_{4} \mathrm{O}_{2}$ uniti ai vertici direttamente tra loro ed anche da tetraedri di ione solfato. La thermessaite si collega per composizione chimica, ma non per struttura, ad un gruppo di alluminofluoruri con altri anioni, come la rara stenonite $\mathrm{Sr}_{2}\left[\mathrm{AlF}_{5} \mathrm{CO}_{3}\right]$ e la ancora più rara bøggildite $\mathrm{Sr}_{2} \mathrm{Na}_{2}\left[\mathrm{Al}_{2} \mathrm{~F}_{9} \mid \mathrm{PO}_{4}\right]$, che provengono unicamente dalla Groenlandia, la creedite $\mathrm{Ca}_{3}\left[\mathrm{Al}_{2}(\mathrm{~F}, \mathrm{OH})_{10} \mid \mathrm{SO}_{4}\right] \cdot 2 \mathrm{H}_{2} \mathrm{O}$, presente nel mondo in diverse località, tra cui la Sardegna, e la chukhrovite- $(\mathrm{Y}) \mathrm{Ca}_{3}(\mathrm{Y}, \mathrm{Ce})\left[\mathrm{Fl}\left(\mathrm{AlF}_{6}\right)_{2} \mid \mathrm{SO}_{4}\right]$. $12 \mathrm{H}_{2} \mathrm{O}$, quest'ultima soprattutto della Russia. Ad eccezione della sola gearksutite, tuttavia, non si sono osservati altri fluoruri complessi comuni a Vulcano ed alle altre località; al Vesuvio, invece, si è trovata la ralstonite $\mathrm{Na}_{\mathrm{x}} \mathrm{Mg}_{\mathrm{x}} \mathrm{Al}_{2-\mathrm{x}}(\mathrm{F}, \mathrm{OH})_{6} \cdot \mathrm{H}_{2} \mathrm{O}$, un altro minerale caratteristico di Ivigtut (CAROBBI \& CIPRIANI, 1952), e - come scoperta recentissima ancora la gearksutite (CAMPOSTRINI, 2012, comunicaz. personale). In vista della scarsa volatilità dei fluoruri di alluminio, tutti gli allumino- 
fluoruri vulcanici non sono stati depositati direttamente dai vapori, ma assai probabilmente derivano dall'attacco delle rocce alluminifere effettuato dagli acidi fluoridrico e solforico alla bocca delle fumarole.

\section{L'ammonio}

Nelle fumarole di Vulcano sono presenti notevoli quantità di sali ammoniacali, tra cui soprattutto il cloruro ammonico, che nei secoli passati è stato a volte così abbondante da essere oggetto di sfruttamento economico. Vi è tuttavia una ricchezza insolita di altre specie: accanto a a quelle più comuni, come la mascagnite $\left(\mathrm{NH}_{4}\right)_{2} \mathrm{SO}_{4}$, ve ne sono altre molto più rare ed interessanti, e spesso uniche o quasi al mondo. In effetti, alla Fossa è presente la rara godovikovite $\mathrm{NH}_{4}(\mathrm{Al}, \mathrm{Fe}) \mathrm{SO}_{4}$, che fu scoperta in varie località, tuttavia sinora soltanto come prodotto della combustione di miniere di carbone (SHCHERBAKOVA et al., 1988), nonché la larderellite $\left(\mathrm{NH}_{4}\right) \mathrm{B}_{5} \mathrm{O}_{7}(\mathrm{OH})_{2} \cdot \mathrm{H}_{2} \mathrm{O}$ che era stata ritenuta unica di Larderello. Tra i sali di ammonio osservati solo a Vulcano, oltre alla barberiite $\mathrm{NH}_{4} \mathrm{BF}_{4}$ (GARAVELLI \& VURRO, 1994), si considerino anche la pyracmonite $\left(\mathrm{NH}_{4}\right)_{3} \mathrm{Fe}\left(\mathrm{SO}_{4}\right)_{3}$, la panichiite $\left(\mathrm{NH}_{4}\right)_{2} \mathrm{SnCl}_{6}$, la brontesite $\left(\mathrm{NH}_{4}\right)_{3} \mathrm{PbCl}_{5}$ (DEMARTIN et al., 2009ab), l'adranosite $\mathrm{NaAl}_{2}\left(\mathrm{NH}_{4}\right)_{4}\left(\mathrm{SO}_{4}\right)_{4} \mathrm{Cl}(\mathrm{OH})_{2}$, di struttura molto complessa e l'argesite $\left(\mathrm{NH}_{4}\right)_{7} \mathrm{Bi}_{3} \mathrm{Cl}_{16}$ (DEMARTIN et al., 2012); anche in questi casi, come per i fluoruri, molti di questi minerali possono essere a buon diritto considerati come esempi di complessi alogenati di vari elementi (ferro, piombo, stagno, bismuto, alluminio), così stabili da sfidare la tendenza a decomporsi in ammoniaca ad alta temperatura.

In alcune di queste specie può giocare la sostituzione dell'ammonio con il potassio, in vista della notevole vicinanza dei raggi ionici. Un esempio evidente è quello della kremersite $\left(\mathrm{NH}_{4}\right)_{2} \mathrm{FeCl}_{5} \cdot \mathrm{H}_{2} \mathrm{O}$, un minerale questo che a Vulcano sembra essere molto più abbondante dell'eritrosiderite, e cioè del corrispondente termine potassico $\mathrm{K}_{2} \mathrm{FeCl}_{5} \cdot \mathrm{H}_{2} \mathrm{O}$, con cui è isomorfa e che invece è molto più comune altrove, come ad esempio al Vesuvio; limitate sostituzioni dell'ammonio con il potassio si osservano anche in altri minerali, come nella pyracmonite $\left(\mathrm{NH}_{4}\right)_{3} \mathrm{Fe}\left(\mathrm{SO}_{4}\right)_{3}$, sebbene questa sia di fatto praticamente esclusiva per l'ammonio, in vista della formazione di deboli legami ad idrogeno (N-H......... $)$ con gli atomi di ossigeno degli ioni solfato che gli ioni $\mathrm{NH}_{4}{ }^{+}$possono formare, a differenza di $\mathrm{K}^{+}$(DEMARTIN et al., 2010a). Ed infatti non ne è noto l'equivalente puro di potassio, né naturale, né sintetico. 
Esiste inoltre un problema assai importante per quanto riguarda l'origine dell'ammonio nelle fumarole vulcaniche, un problema questo che risulta ancora oggi alquanto controverso. Dato che l'azoto non è un componente diffuso nelle "normali" rocce, soprattutto in passato, e già dai primi decenni dell'Ottocento, qualcuno (ABICH, 1835) era giunto a supporre che l'ammoniaca derivasse dalla decomposizione di vegetali od altro materiale organico depositato in qualche modo alla bocca delle fumarole, un'ipotesi questa sostenuta anche da famosi chimici di allora come BUNSEN e da geologi come PILLA e RANIERI. Tuttavia, come in altre località, è veramente arduo supporre che attorno alla bocca delle fumarole della Fossa vi siano tracce di materiali organici, e pertanto l'ammoniaca deve essere già presente nei gas vulcanici, un'ipotesi questa già sostenuta dall'ANGELOT nel 1841 e come del resto è dimostrato dalle loro analisi. Una supposizione suggestiva è che in effetti l'ammoniaca, come gli altri gas almeno in parte, derivi dalla degassazione del materiale profondo dell'interno della Terra, che dura tuttora; un forte indizio a favore di una simile ipotesi è la composizione isotopica dell'elio contenuto in questi gas delle fumarole, dove il rapporto tra la concentrazione dell'isotopo ${ }^{3} \mathrm{He}$ e quella corrispondente dell'isotopo molto più comune ${ }^{4} \mathrm{He}$ $\left(6,95 \times 10^{-6}\right)$ supera notevolmente quello $\left(1,4 \times 10^{-6}\right)$ osservato nell'atmosfera terrestre (MARTI in MAZOR et al., 1988; ITALIANO \& NUCCIO, 1996; ITALIANO et al., 1998); un'altra conferma é l'esistenza, in non poche formazioni vulcaniche, di minerali di ammonio come veri e propri componenti delle rocce, quali miche (tobelite, gumbelite), e persino feldspati (buddingtonite).

\section{Il tallio}

Non meno interessante è il comportamento del tallio: questo raro elemento fu notato in tracce nello "zolfo" delle isole Eolie già dal CROOKES nei suoi lavori di ricerca di questo nuovo elemento, da lui appena scoperto per mezzo dell'analisi spettroscopica, in tutti i minerali allora conosciuti.

Dal punto di vista chimico, il tallio possiede proprietà davvero singolari, per cui qualcuno lo ha chiamato l' "ornitorinco" dei metalli. Lo strano comportamento chimico del tallio è dovuto, secondo SIDGWICK e molti altri chimici, al cosiddetto "doppietto inerte" dei cosiddetti elettroni del sottostrato $6 \mathrm{~s}$ che si manifesta in questi elementi pesanti (come anche per il piombo) e che lo fa assomigliare ad un sottostrato esterno 
completo come quello $1 s$ dell'elio. Di conseguenza, mentre nei composti covalenti il tallio assomiglia soprattutto all'argento ed un poco meno al piombo, i composti a carattere ionico del tallio e del piombo (ovvero, per i minerali, quelli non solforati) gli elettroni ancora più esterni $6 p$ (uno per $\mathrm{Tl}$ e due per $\mathrm{Pb}$, che è l'elemento che lo segue immediatamente in ordine di numero atomico) sono invece simili rispettivamente a quelli più esterni $s$ dei metalli alcalini od alcalino-terrosi. Ne segue che molti composti non solforati del tallio, piuttosto che a quelli del piombo e dell'argento, assomiglino invece non poco a quelli di $\mathrm{K}, \mathrm{Rb}$ e Cs, ed anche dell'ammonio $\mathrm{NH}_{4}$ per via della somiglianza dei raggi ionici. Per esempio, caratteristica è la forte alcalinità dell'idrossido $\mathrm{TlOH}$, che è solubile nell'acqua e non assomiglia certo a quello di nessun altro metallo pesante; anche il carbonato $\mathrm{Tl}_{2} \mathrm{CO}_{3}$ è notevolmente solubile nell'acqua, quasi come fosse un carbonato alcalino.

Solfuri di tallio e bismuto, od anche di tallio e piombo furono osservati già nel 2002 da AFRICANO et al. nelle fumarole del vulcano Satsuma-Iwojima in Giappone. Nelle fumarole di Vulcano, invece, non sono stati scoperti finora solfuri notevolmente talliferi, ma qui ed altrove il tallio tende piuttosto a formare alogenuri, in relazione alla straordinaria stabilità di questi composti e dell'abbondanza di alogeni (soprattutto cloro) nei fluidi. Nel 1906 ZAMBONINI menziona la presenza di quantità minori di tallio nelle silviti $(\mathrm{KCl})$ vesuviane, ed BRUN (1917) prese in esame lo studio di minerali contenenti elementi più rari, tra cui il tallio, nei prodotti fumarolici; tuttavia la scoperta di minerali veri e propri di questo metallo nelle fumarole dei vulcani è molto più recente. La prima scoperta di questi si deve a FULIGNATI e SBRANA che osservarono nel 1998 per la prima volta la presenza di alogenuri di tallio a Vulcano, una scoperta questa confermata qualche anno dopo da ROBERTS et al. (2006) che scoprirono una varietà bromifera di lafossaite $(\mathrm{TlCl})$ proprio nel cratere della Fossa che ne è la località-tipo (da cui il nome del minerale). Contemporaneamente, un esemplare significativo, non bromifero, di questa specie, raccolto nel 1906 al Vesuvio assieme a dimorfina $\left(\mathrm{As}_{4} \mathrm{~S}_{3}\right)$, fu osservato da CAMPOSTRINI, testimoniando così una maggiore diffusione di alogenuri di tallio nelle fumarole vulcaniche di quanto non fosse noto, in accordo con la natura dei prodotti depositati in tubi di quarzo introdotti nelle fumarole, sia nella nostra località che in altri vulcani, come quelli della Kamchatka e delle isole Kurili. A questo proposito, infatti, ZELENSKI \& BORTNIKOVA nel 2005 scoprirono un cloruro di tallio e piombo $\mathrm{PbTl}_{3}(\mathrm{Cl}, \mathrm{Br}, \mathrm{I})_{5}$ nelle fumaro- 
le del vulcano Mutnovsky, oltre ad altri alogenuri talliferi non ancora sufficientemente studiati, tra cui lo ioduro TlI ed un minerale probabilmente identico all' hephaistosite $\mathrm{TlPb}_{2} \mathrm{Cl}_{5}$, questi ultimi rilevati tuttavia soltanto come sublimati entro tubi di quarzo.

Alla scoperta della lafossaite nella nostra isola seguì ben presto quella di altri cloruri di tallio, quali l'hephaistosite $\mathrm{TlPb}_{2} \mathrm{Cl}_{5}$ (CAMPOSTRINI et al., 2008) e la steropesite $\mathrm{Tl}_{3} \mathrm{BiCl}_{6}$ (DEMARTIN et al., 2009): di tutti questi Vulcano resta la località-tipo, assieme ad un numero considerevole di bromuri e ioduri potenzialmente nuovi e tuttora in corso di studio (CAMPOSTRINI et al., 2011).

In relazione a quanto si è detto sopra per i minerali non solforati di tallio, che assomigliano ai composti di metalli alcalini, la struttura della lafossaite è simile a quella del cloruro di cesio e del cloruro d'ammonio; per l'hephaistosite si ha una notevole somiglianza (probabilmente isomorfismo) con il corrispondente termine di potassio $\mathrm{KPb}_{2} \mathrm{Cl}_{5}$ o challacolloite (Mitolo et al., 2009). Esistono anche relazioni, per quanto non così strette, tra la steropesite $\mathrm{Tl}_{3} \mathrm{BiCl}_{6}$ ed i cloruri (sintetici) di cesio e bismuto, od anche di cesio, potassio e bismuto $\mathrm{Cs}_{3} \mathrm{BiCl}_{6} \mathrm{e}$ $\mathrm{CsK}_{2} \mathrm{BiCl}_{6}$ (DEMARTIN et al., 2009), che si possono a loro volta considerare come veri e propri complessi (esaclorobismutati).

Lo stagno

Oltre all'interesse per il contenuto di ammonio, la panichiite $\left(\mathrm{NH}_{4}\right)_{2} \mathrm{SnCl}_{6}$ (DEMARTIN et al., 2009a): è l'unico cloruro di stagno (4+) sinora trovato in natura, indicando la notevole stabilità dello ione complesso esaclorostannato(IV) $\left(\mathrm{SnCl}_{6}\right)^{2-}$ in essa presente: la sua formazione è quasi certamente legata al trasporto di questo elemento nel vapore sotto forma del tetracloruro $\mathrm{SnCl}_{4}$, molto volatile, e come tale già noto ai chimici sin dal Seicento ("spiritus fumans Libavii"); anche per il solfuro vurroite $\mathrm{Pb}_{20} \mathrm{Sn}_{2}(\mathrm{Bi}, \mathrm{As})_{22} \mathrm{~S}_{54} \mathrm{Cl}_{6}$, che contiene pure stagno (GARAVELLI et al., 2005), è molto verosimile supporre che questo elemento sia stato depositato dal tetracloruro.

\section{Oro, argento e tellurio}

Nelle fumarole di Vulcano si trovano anche tracce di metalli preziosi: un esempio importante è quello dell'oro nativo, scoperto da FULIGNATI \& SBRANA (1998) assieme a tellurio, un altro metallo questo, 
la cui presenza fu già intravista dal CossA (1898) che accompagna spesso l'oro, come del resto anche il bismuto. L'argento sembra invece (come il rame) particolarmente scarso, al punto che persino le lillianiti di Vulcano non sono argentifere come le altre. Molto recentemente, l'argento è stato individuato (CAMPOSTRINI et al., 2011) come elemento essenziale in uno strano ed interessantissimo solfato, denominato provvisoriamente UKIfo13-(SO:Ca,K,Ag,Sr,Ce) che contiene anche terre rare.

\section{Minerali non solforati del piombo}

A parte l'interesse per le strutture assai complesse dei solfuri, negli alogenuri (presenti in abbondanza nelle fumarole di Vulcano) il piombo manifesta un notevole interesse chimico nei composti ionici a causa del doppietto inerte di elettroni $6 \mathrm{~s}$ che lo fa assomigliare ai metalli alcalinoterrosi ed alcalini od addirittura all'ammonio per via della somiglianza del raggio ionico. Ad alta temperatura queste somiglianze si manifestano nel disordine strutturale $\mathrm{NH}_{4}-\mathrm{Pb}$ della brontesite $\left(\mathrm{NH}_{4}\right)_{3} \mathrm{PbCl}_{5}$ (DEMARTIN et al., 2009c); qualcosa di simile deve verificarsi anche per la pseudocotunnite $\mathrm{K}_{2} \mathrm{PbCl}_{4}$ (CAMPOSTRINI et al., 2011), una specie che è particolarmente frequente a Vulcano insieme alla challacolloite, e che mostra la particolarità di fornire cristalli incolori e limpidi ad alta temperatura, ma che diventano abbastanza rapidamente opachi se portati a temperatura ambiente. Una tale situazione è stata effettivamente osservata almeno per la transizione ordine-disordine che avviene ad alta temperatura per il composto sintetico $\mathrm{KPb}_{2} \mathrm{Cl}_{5}$ corrispondente alla challacolloite (VELÁZQUEZ et al., 2006), e qualcosa di simile si verifica anche tra il tallio ed il piombo nel composto sintetico $\mathrm{Tl}_{3} \mathrm{PbCl}_{5}$, la cui struttura è fortemente disordinata (SKARSTAD et al., 1979); l'apparente geminazione osservata dagli scopritori nel cristallo da loro studiato di hephaistosite potrebbe in realtà essere ascritto anche ad un parziale disordine di questo tipo. Nella brontesite si manifesta anche una competizione termodinamica tra il disordine (favorito dall'alta temperatura) e la dissociazione in ammoniaca volatile: questa competizione rende particolarmente ridotto l'intervallo di stabilità della specie.

\section{I solfati}

A differenza delle fumarole del cratere principale, solfati di metalli comuni sono invece presenti con molto maggiore abbondanza soprat- 
tutto nelle fumarole di bassa temperatura della Baia di Levante, e si formano prevalentemente per azione dell'acido solforico (derivato a sua volta dall'ossidazione all'aria di gas solforati fumarolici) sulle rocce circostanti, che sono in genere ricche di alluminio e di magnesio, non solo, ma anche di metalli alcalini $(\mathrm{Na}, \mathrm{K})$ e di ferro. Tra questi prodotti, assai frequente è soprattutto l'alunogeno $\mathrm{Al}_{2}\left(\mathrm{SO}_{4}\right)_{3} \cdot 17 \mathrm{H}_{2} \mathrm{O}$ : questo minerale è stato assai probabilmente la vera fonte naturale dell'allume $\mathrm{KAl}\left(\mathrm{SO}_{4}\right)_{2} \cdot 12 \mathrm{H}_{2} \mathrm{O}$, che veniva prodotto per lo più artificialmente, ma che in quantità subordinata si trova pure in natura; un raro solfato di alluminio è invece la zaherite $\mathrm{Al}_{12}\left[(\mathrm{OH})_{26} \mathrm{l}\left(\mathrm{SO}_{4}\right)_{5}\right] \cdot 20 \mathrm{H}_{2} \mathrm{O}$.

Si ha poi tutta una serie di termini ferriferi, tra cui soprattutto abbondante é la metavoltina $(\mathrm{K}, \mathrm{Na})_{8} \mathrm{Fe}^{2+} \mathrm{Fe}^{3+}{ }_{6}\left(\mathrm{SO}_{4}\right)_{12} \mathrm{O}_{2} \cdot 18 \mathrm{H}_{2} \mathrm{O}$, che non raramente è accompagnata dalla voltaite $\mathrm{K}_{2} \mathrm{Fe}_{5}{ }^{2+} \mathrm{Fe}_{3}{ }^{3+} \mathrm{Al}\left(\mathrm{SO}_{4}\right)_{12}$ $\mathrm{O}_{2} \cdot 18 \mathrm{H}_{2} \mathrm{O}$; in questa specie sono importanti le sostituzioni $\mathrm{Mg} \mathrm{Fe}^{2+}$, che possono portare anche alla pertlikite, che è il termine corrispondente con prevalenza di magnesio, così come si osserva nelle altre coppie mohrite $\left(\mathrm{NH}_{4}\right)_{2} \mathrm{Fe}\left(\mathrm{SO}_{4}\right)_{2} \cdot 6 \mathrm{H}_{2} \mathrm{O}$ - boussingaultite $\left(\mathrm{NH}_{4}\right)_{2} \mathrm{Mg}\left(\mathrm{SO}_{4}\right)_{2}$ $\cdot 6 \mathrm{H}_{2} \mathrm{O}$ o halotrichite-pickeringite $\mathrm{Fe}^{2+} \mathrm{Al}_{2}\left(\mathrm{SO}_{4}\right)_{4} \cdot 22 \mathrm{H}_{2} \mathrm{O}-\mathrm{MgAl}_{2}\left(\mathrm{SO}_{4}\right)_{4}$ $\cdot 22 \mathrm{H}_{2} \mathrm{O}$.

A Vulcano si osservano anche altre sostituzioni cristallochimiche importanti, come quella di $\mathrm{Cu}$ con $\mathrm{Mg}$ nella rara magnesioaubertite $(\mathrm{Cu}, \mathrm{Mg}) \mathrm{Al}\left(\mathrm{SO}_{4}\right)_{2} \mathrm{Cl} \cdot 14 \mathrm{H}_{2} \mathrm{O}$ (GEBHARD et al., 1988; GARAVELLI et al., 1996), e quella del ferro (3+) con l'alluminio nella coquimbite $\left(\mathrm{Fe}, \mathrm{Al}_{2}\right)_{2}\left(\mathrm{SO}_{4}\right)_{3} \cdot 9 \mathrm{H}_{2} \mathrm{O}$ : quest'ultima sostituzione è probabilmente all'origine del malinteso della "millosevichite", di cui almeno alcuni dei campioni originari non sono altro che varietà fortemente alluminifere di coquimbite, ma si ha almeno un'altra specie distinta, scoperta molto recentemente: l'aluminocoquimbite $\mathrm{AlFe}\left(\mathrm{SO}_{4}\right)_{3} \cdot 9 \mathrm{H}_{2} \mathrm{O}$, che corrisponde ad una ben definita variazione strutturale (DEMARTIN et al., 2010e). Questa variazione é legata alla tendenza più marcata del ferro $(3+)$ di legarsi ai solfati rispetto a quella dell'alluminio, che preferisce coordinarsi all'acqua. In queste fumarole è stata osservata anche la copiapite $\mathrm{Fe}^{2+} \mathrm{Fe}_{4}{ }^{3+}\left(\mathrm{SO}_{4}\right)_{6}(\mathrm{OH})_{2} \cdot 20 \mathrm{H}_{2} \mathrm{O}$, una specie molto comune soprattutto alla superficie delle miniere di ferro, ma che qui sembra essere invece subordinata alla metavoltina.

La paragenesi di specie interessanti contenenti ferro e potassio (metavoltina e talora krausite $\mathrm{KFe}^{3+}\left(\mathrm{SO}_{4}\right)_{2} \cdot \mathrm{H}_{2} \mathrm{O}$ ) assieme a minerali di solo ferro ed alluminio (coquimbite, halotrichite), in relazione ad attività fumaroliche non troppo intense ed alla conseguente azione di acido 
solforico su rocce ricche di ferro e - particolarmente - di potassio ricalca quanto si è già osservato in altre località di tipo vulcanico, tra cui quelle della California (Calico Hills) ed alla solfatara di Pozzuoli (FerRaris e Gramaccioli, 1971). Come a Pozzuoli, anche a Vulcano è stata recentemente scoperta la rarissima yavapaiite $\mathrm{KFe}^{3+}\left(\mathrm{SO}_{4}\right)_{2}$. Quest'ultima specie, che è anidra, scoperta originariamente in Arizona, è legata ad un ambiente di formazione non prettamente vulcanico, di temperatura piuttosto elevata e molto ricco di acido solforico, mentre il contrario è vero per un altro solfato di ferro e di potassio, la goldichite $\mathrm{KFe}^{3+}\left(\mathrm{SO}_{4}\right)_{2} \cdot 4 \mathrm{H}_{2} \mathrm{O}$, scoperta originariamente nelle miniere di San Rafael Swell nell'Utah, e che è relativamente comune a Pozzuoli, ma non è stata ancora trovata né a Vulcano, né in California. Anche qui l'assenza di equivalenti (almeno naturali) di alluminio per tutte queste specie evidenzia una differenza apprezzabile rispetto al ferro.

Interessante è la presenza di solfati che contengono anche cloro: come l' aiolosite $\mathrm{Na}_{4} \mathrm{Bi}\left(\mathrm{SO}_{4}\right)_{3} \mathrm{Cl}$, che abbiamo già visto. Di questi solfati-cloruri, oltre alla magnesioaubertite, osserviamo la rarissima adranosite $\mathrm{NaAl}_{2}\left(\mathrm{NH}_{4}\right)_{4}\left(\mathrm{SO}_{4}\right)_{4} \mathrm{Cl}(\mathrm{OH})_{2}$ (DEMARTIN et al., 2010b) e la vlodavetsite $\mathrm{AlCa}_{2}\left(\mathrm{SO}_{4}\right)_{2} \mathrm{~F}_{2} \mathrm{Cl} \cdot 4 \mathrm{H}_{2} \mathrm{O}$, della quale Vulcano risulta essere il secondo ritrovamento al mondo, dopo la località tipo del vulcano Tolbachik in Kamchatka (VERGASOvA et al., 1995).

Tra i minerali sodiferi, abbondante è soprattutto la tamarugite $\mathrm{NaAl}\left(\mathrm{SO}_{4}\right)_{2} \cdot 6 \mathrm{H}_{2} \mathrm{O}$ (GARAVELLI et al., 1996). Molto subordinati, ma presenti, sono altri minerali di sodio, come la blödite $\mathrm{Na}_{2} \mathrm{Mg}\left(\mathrm{SO}_{4}\right)_{2} \cdot 4 \mathrm{H}_{2} \mathrm{O}$, la mirabilite $\mathrm{Na}_{2}\left(\mathrm{SO}_{4}\right) \cdot 10 \mathrm{H}_{2} \mathrm{O}$, la thenardite $\mathrm{Na}_{2} \mathrm{SO}_{4}$ e la sideronatrite $\mathrm{Na} 2 \mathrm{Fe}\left(\mathrm{SO}_{4}\right)_{2}(\mathrm{OH}) \cdot 3 \mathrm{H}_{2} \mathrm{O}$. Tra le poche specie contenenti ammonio presenti in queste fumarole della Baia di Levante, ricordiamo ancora la boussingaultite $\left(\mathrm{NH}_{4}\right)_{2} \mathrm{Mg}\left(\mathrm{SO}_{4}\right)_{2} \cdot 4 \mathrm{H}_{2} \mathrm{O}$, mentre anche in quelle del cratere della Fossa é stata osservato l'allume d'ammonio tschermigite $\mathrm{NH}_{4} \mathrm{Al}\left(\mathrm{SO}_{4}\right)_{2} \cdot 12 \mathrm{H}_{2} \mathrm{O}$ (CAMPOSTRINI et al., 2011); tra i minerali di calcio è presente invece in abbondanza il gesso $\mathrm{CaSO}_{4} \cdot 2 \mathrm{H}_{2} \mathrm{O}$.

Mentre i minerali di rame sono in pratica del tutto assenti nelle fumarole della cima del vulcano La Fossa, in quelle della Baia di Levante è abbastanza frequente il comune solfato di rame o calcantite $\mathrm{CuSO}_{4} \cdot 5 \mathrm{H}_{2} \mathrm{O}$; molto più interessante è la presenza della magnesioaubertite $(\mathrm{Cu}, \mathrm{Mg}) \mathrm{Al}\left(\mathrm{SO}_{4}\right)_{2} \mathrm{Cl} \cdot 14 \mathrm{H}_{2} \mathrm{O}$ (GEBHARD et al., 1988), una specie trovata unicamente a Vulcano che non si trova tuttavia nelle stesse fumarole che hanno fornito la calcantite. 
Altri dati interessanti riguardano la sostituzione dei metalli alcalini (potassio con il cesio o con l'ammonio, od anche con il tallio). Che ad esempio l'allume di Vulcano sia cesifero è noto dagli studi del CossA della fine dell'Ottocento che non sono di molto posteriori alla stessa scoperta del cesio. Qui tuttavia non è ben chiaro se l'arricchimento in questo metallo non dipenda piuttosto dall'effetto di ricristallizzazioni effettuate sul materiale (non sempre del tutto naturale, in quanto lo stesso Cossa nel 1881 parla di aggiunte di ceneri di sarmenti di viti, o di cloruro ammonico); un parallelo riguarda l'avogadrite $\mathrm{KBF}_{4}$, di cui l'unico campione sinora trovato a Vulcano (CAMPOSTRINI et al., 2011) non é sensibilmente cesifero, a differenza di alcuni esemplari-tipo vesuviani, scoperti nel 1926 dallo ZAMBONINI. Tuttavia, non si può del tutto escludere il caso che il materiale del Vesuvio sia stato di fatto ricristallizzato, o naturalmente per azione dell'acqua sui prodotti fumarolici, od anche, almeno in parte, artificialmente. Della serie challacolloitehephaistosite, ove tallio e potassio si sostituiscono a vicenda, od anche della sostituzione ammonio-potassio nell'eritrosiderite-kremersite e nella pyracmonite è stato già detto.

Un solfato molto interessante, che contiene anche notevoli quantità di fluoruro ed anche dello ione idrogenosolfato, assai raro questo in natura, é la cossaite $\mathrm{Mg}_{0,5} \mathrm{Al}_{6}\left(\mathrm{SO}_{4}\right)_{6}\left(\mathrm{HSO}_{4}\right) \mathrm{F}_{6} 36 \mathrm{H}_{2} \mathrm{O}$, di scoperta assai recente (DEMARTIN et al., 2011b).

\section{Il boro}

A Vulcano sono infine presenti parecchi minerali di boro: tra questi vi è soprattutto l'acido borico o sassolite $\mathrm{H}_{3} \mathrm{BO}_{3}$, una specie questa tipica come abbondante prodotto delle fumarole della Fossa e per la cui presenza la nostra isola è una località classica, nota da parecchio tempo. Di scoperta assai recente e molto interessante è invece l'acido metaborico $\mathrm{HBO}_{2}$ o metaborite, come secondo ritrovamento al mondo (CAMPOSTRINI et al., 2011): questa specie si trova pure, tuttavia solo come grande rarità, in alcune fumarole, associata a molta sassolite. Tuttavia, nelle località tipo (cupola salifera della valle di Ak-sa in Russia e lago Inder nel Kazakhstan) la metaborite è una specie dei giacimenti saliferi, ove è presente associata a molto salgemma (halite) e vari borati e dove, per la forte concentrazione salina, l'attività dell'acqua è relativamente bassa ma la temperatura non è particolarmente alta. A Vulcano, invece, la metaborite si è formata quale prodotto di deposizione dei vapori delle 
fumarole ma la concentrazione (più precisamente, l'attività) dell' acqua deve essere inferiore a quella che permette la formazione e la deposizione della sassolite, un prodotto questo anche volatile in presenza di vapore. Qualche anno fa a Vulcano è stata scoperta anche la clinometaborite (DEMARTIN et al., 2011), una modificazione monoclina dell'acido metaborico che era nota sinora soltanto come composto artificiale.

Oltre all'acido borico ed ai fluoroborati recentemente scoperti (barberiite, avogadrite, knasibfite: vedi sopra) che non escluderebbero la presenza nei gas delle fumarole del trifluoruro $\mathrm{BF}_{3}$, il boro nei prodotti fumarolici della Fossa forma anche veri e propri borati, di scoperta assai recente (CAMPOSTRINI et al., 2011). Oltre alla larderellite $\left(\mathrm{NH}_{4}\right) \mathrm{B}_{5} \mathrm{O}_{7}(\mathrm{OH})_{2} \cdot \mathrm{H}_{2} \mathrm{O}$, abbiamo anche la rara santite $\mathrm{K}\left[\mathrm{B}_{5} \mathrm{O}_{6}(\mathrm{OH})_{4}\right]$. $2 \mathrm{H}_{2} \mathrm{O}$, che fu pure scoperta a Larderello, nei soffioni boraciferi (MERLINO \& SARTORI, 1970).

\section{CONCLUSIONI}

L'Isola di Vulcano si è rivelata una località mineralogica di rilevanza mondiale, la cui ricchezza mineralogica la pone, dopo il Vesuvio, al secondo posto tra le località italiane per numero di specie descritte per la prima volta e che offre parecchi interessanti esempi alla chimica. Molti di questi minerali sono il risultato della deposizione di molecole volatili come tali (zolfo, cloruro ferrico); altri derivano dalla reazione dell'ammoniaca con $\mathrm{HCl}, \mathrm{HF}, \mathrm{BF}_{3}, \mathrm{SiF}_{4}$, ecc.; altri ancora (soprattutto i cloruri complessi, o gli stessi ossidi od ossicloruri) sono invece prodotti della reazione di cloruri metallici volatili $\left(\mathrm{Fe}_{2} \mathrm{Cl}_{6}, \mathrm{BiCl}_{3}, \mathrm{PbCl}_{2}\right.$, $\mathrm{SnCl}_{4}$, ecc.) con acqua od ammoniaca, od anche tra questi stessi cloruri ed idrogeno solforato.

Gli equilibri termodinamici che ne conseguono sono particolarmente complicati ed interessanti, sempre ammesso che di equilibri si tratti. Si tenga presente che la formazione di complessi più studiata nei laboratori avviene da reazioni in fase liquida (soprattutto acquosa) piuttosto che in fase gassosa.

Altri minerali, soprattutto quelli alluminiferi, derivano sicuramente dall'attacco delle rocce circostanti, e la situazione si complica ulteriormente in presenza di vapori di ammoniaca.

L'arricchimento in bromo e iodio non può che spiegarsi tramite arricchimenti nei vapori stessi, come residuo di degassazione dei mate- 
riali interni profondi; questo arricchimento può essere spinto anche in fase finale, all'atto della deposizione dei sublimati.

Uno studio sistematico comparativo dei minerali di Vulcano con quelli del Vesuvio, ha permesso di osservare tra i due vulcani notevoli analogie ed anche sostanziali differenze. Di particolare interesse è la presenza nelle fumarole di entrambi i vulcani di alcuni fluoruri complessi (hieratite, avogadrite, thermessaite) e cloruri di metalli pesanti (cotunnite, pseudocotunnite e challacolloite), tra cui anche minerali di tallio (lafossaite). D'altro canto è particolarmente evidente il fatto che, mentre al Vesuvio sono presenti numerosi minerali contenenti rame, questi invece a Vulcano mancano quasi completamente.

Viceversa, nelle fumarole di Vulcano sono particolarmente abbondanti i minerali contenenti bismuto (specialmente solfuri e solfosali, ma anche alogenuri) che invece al Vesuvio sono del tutto assenti; inoltre, le specie tallifere sinora scoperte sembrano essere molte di più.

Purtroppo, un confronto esauriente tra i due vulcani rimane difficile, dato che al Vesuvio l'attività delle fumarole non è confrontabile con quella che si aveva prima dell'ultima eruzione del 1944, e pertanto non si può escludere che parecchi dei minerali di aspetto incospicuo (vedi ad esempio gli alogenuri di tallio e diversi fluoruri complessi), che soltanto oggi sarebbe stato possibile indiuviduare e studiare adeguatamente con mezzi moderni, siano di fatto sfuggiti in gran parte all'attenzione ed alla raccolta; esempi a questo proposito vengono forniti dalle scoperte della lafossaite e della thermessaite effettuate su vecchi campioni vesuviani proprio in questi ultimi anni, in occasione di un sistematico raffronto con Vulcano.

Infine, una differenza saliente con il Vesuvio è la quasi totale mancanza a Vulcano di quei minerali caratteristici dei proietti che, con la loro bellezza hanno reso famoso nel mondo il vulcano campano.

Vulcano, tenendo fede al suo nome, sembra essere una vera fucina di specie nuove e, viste le continue e numerose scoperte, si può sicuramente affermare che il lavoro da svolgere negli anni a venire rimane ancora molto. A questo proposito, si auspica quindi di riuscire a caratterizzare esaurientemente molte delle decine di specie sicuramente nuove ed assai interessanti che non sono state ancora ufficialmente approvate dalla comunità dei mineralogisti per mancanza di dati adeguati. Una simile potenzialità di nuove scoperte mineralogiche sembra pertanto essere unica almeno in Europa, od addirittura al mondo, con l'unica riserva che riguarda i famosi vulcani della Kamchatka e delle isole Kurili. 


\section{RIFERIMENTI BIBLIOGRAFICI}

Africano, F., Van Rompaey, G., Bernard, A. \& Le Guern, F. (2002): Deposition of trace elements from high temperature gases of Satsuma-Iwojima volcano. Earth Planets Space 54, 275-286.

BERNAUER, F. (1941): Eine Gearksutit-Lagerstätte auf der Insel Vulcano. Zeitschrift der Deutschen Geologischen Gesellschaft 93, 65-80.

Borodaev, Y.S, Garavelli, A., Kuzmina, O.V., Mozgova, N.N., Organova, N.I., TrubKin, N.V., VurRo, F. (1998): Rare sulfosalts from Vulcano, Aeolian Islands, Italy. I. Se-bearing kirkiite, $\mathrm{Pb}_{10}(\mathrm{Bi}, \mathrm{As})_{6}(\mathrm{~S}, \mathrm{Se})_{19}$. Can. Mineral. 36, 1105-1114.

Borodaev, Y.S., Garavelli, A., Garbarino, C., Grillo, S., Organova, N.I., Trubkin, N.V. \& Vurro, F. (2000): Rare sulfosalts from Vulcano, Aeolian Islands, Italy. III. Wittite and cannizzarite. Can. Mineral. 38, 23-34.

Borodaev, Y.S., Garavelli, A., Garbarino, C., Grillo, S.M., Mozgova, N.N., UsPensKaya, T.Y. \& VurRo, F. (2001): Rare sulfosalts from Vulcano, Aeolian Islands, Italy. IV. Lillianite. Can. Mineral. 39, 1383-1396.

Borodaev, Y.S., Garavelli, A., Garbarino, C., Grillo, S.M., Mozgova, N.N., PaAr, W.H., TopA, D. \& Vurro, F. (2003): Rare sulfosalts from Vulcano, Aeolian Islands, Italy. V. Selenian heyrovskyite. Can. Mineral. 41, 429-440.

BRun, A. (1917): Contribution à la connaissance de la minéralogie du bore, du lithium et du thallium dans l'exhalation volcanique. Bull.Soc.Franç,Minéral. 31, 107-110.

Campostrini, I., Demartin, F., Gramaccioli, C.M. \& Orlandi, P. (2008): Hephaistosite, $\mathrm{TlPb}_{2} \mathrm{Cl}_{5}$, a new thallium mineral species from La Fossa crater, Vulcano, Aeolian Islands, Italy. Can. Mineral. 46, 701-708.

Campostrini, I., Demartin, F., Gramaccioli, C.M. \& Russo, M. (2011) Vulcano. Tre secoli di mineralogia. Associazione Micro-mineralogica Italiana Ed., Cremona Italy, 344 pages, ISBN 978-88-905541-0-0.

CAROBBI, G. \& Cipriani, C. (1952): Ralstonite e bisolfato sodico (matteuccite) fra i prodotti delle fumarole vesuviane. Rendic.Accad.Naz.le Lincei, ser.8, 12(1), 23-29.

Coradossi, N., Pinarelli, L. \& Bertolini, G.L. (1985): Fluttuazioni del rapporto $\mathrm{Br} / \mathrm{Cl}$ nelle esalazioni fumaroliche di Vulcano (Isole Eolie) e in alcuni prodotti delle loro reazioni. Rendic.Soc.Ital.Min. e Petr. 40, 2, 299-309.

Coradossi, N., Garavelli, A., Salamida, M. \& Vurro, F. (1996) Evolution of Br/Cl ratios in fumarolic salammoniac from Vulcano (Aeolian Islands - Italy). Bulletin of Volcanology, 58, 310-316.

Cossa, A. (1881) Ricerche chimiche sui minerali e roccie dell'Isola di Vulcano. Atti della Reale Accademia dei Lincei, serie III, Rendiconti della Classe di Scienze Fisiche, Matematiche, Naturali, 2, 14 pages.

Cossa, A. (1882a) Sulla hieratite, nuova specie mineralogica. Transunti dell'Accademia dei Lincei, serie III, 6, 141-142. 
CossA, A. (1882b) Sur la Hiératite, espèce minerale nouvelle. Bulletin Societé Française Minéralogie et Cristallographie, 5, 2, 61-62.

Cossa, A. (1884) Hieratit, ein neues Mineral. Zeitschrift für Kristallographie, 8, 305.

Demartin, F., Gramaccioli, C.M., Campostrini, I. \& Orlandi, P. (2008a) Thermessaite $\mathrm{K}_{2}\left[\mathrm{AlF}_{3} \mid \mathrm{SO}_{4}\right]$, a new ino-aluminofluoride-sulfate from La Fossa crater, Vulcano, Aeolian Islands, Italy. Canadian Mineralogist, 46, 693-700.

Demartin, F., Gramaccioli, C.M., Campostrini, I. \& Orlandi, P. (2008b) Demicheleite, BiSBr, a new mineral from La Fossa crater, Vulcano, Aeolian Islands, Italy. American Mineralogist, 93, 1603-1607.

Demartin, F., Campostrini, I. \& Gramaccioli, C.M. (2009a) Panichiite, natural ammonium hexachlorostannate, $\left(\mathrm{NH}_{4}\right)_{2} \mathrm{SnCl}_{6}$, from La Fossa crater, Vulcano, Aeolian Islands, Italy. Canadian Mineralogist, 47, 367-372.

Demartin, F., Gramaccioli, C.M. \& Campostrini, I. (2009b) Steropesite, $\mathrm{Tl}_{3} \mathrm{BiCl}_{6}$, a new thallium bismuth chloride from La Fossa crater, Vulcano, Aeolian Islands, Italy. Canadian Mineralogist, 47, 373-380.

Demartin, F., Gramaccioli, C.M. \& Campostrini, I. (2009c) Brontesite, $\left(\mathrm{NH}_{4}\right)_{3} \mathrm{PbCl}_{5}$, a new product of fumarolic activity from La Fossa Crater, Vulcano, Aeolian Islands, Italy. Canadian Mineralogist, 47, 1237-1243.

Demartin, F., Gramaccioli, C.M. \& Campostrini, I. (2009d) Demicheleite-(Cl), $\mathrm{BiSCl}$, a new mineral from La Fossa Crater, Vulcano, Aeolian Islands, Italy. American Mineralogist, 94, 1045-1048.

Demartin, F., Gramaccioli, C.M. \& Campostrini, I. (2010a) Pyracmonite, $\left(\mathrm{NH}_{4}\right)_{3} \mathrm{Fe}\left(\mathrm{SO}_{4}\right)_{3}$, a new ammonium iron sulfate from La Fossa Crater, Vulcano, Aeolian Islands, Italy. Canadian Mineralogist, 48, 307-313.

Demartin, F., Gramaccioli, C.M. \& CAmpostrini, I. (2010b) Adranosite, $\left(\mathrm{NH}_{4}\right)_{4} \mathrm{NaAl}_{2}\left(\mathrm{SO}_{4}\right)_{4} \mathrm{Cl}(\mathrm{OH})_{2}$, a new ammonium sulfate chloride from La Fossa Crater, Vulcano, Aeolian Islands, Italy. Canadian Mineralogist, 48, 315-321.

Demartin, F., Gramaccioli, C.M. \& Campostrini, I. (2010c) Demicheleite-(I), BiSI, a new mineral from La Fossa Crater, Vulcano, Aeolian Islands, Italy. Min.Mag. 74(1), 141-145.

Demartin, F., Gramaccioli, C.M. \& Campostrini, I. (2010d): Aiolosite, $\mathrm{Na}_{2}\left(\mathrm{Na}_{2} \mathrm{Bi}\right)\left(\mathrm{SO}_{4}\right)_{3} \mathrm{Cl}$, a new sulfate isotypic to apatite from La Fossa Crater, Vulcano, Aeolian Islands, Italy. Am. Mineral. 95, 382-385

Demartin, F., Castellano, C., Gramaccioli, C.M. \& Campostrini, I. (2010e): Aluminocoquimbite, $\mathrm{AlFe}\left(\mathrm{SO}_{4}\right)_{3} \cdot 9 \mathrm{H}_{2} \mathrm{O}$, a new aluminium iron sulphate from Grotta dell'Allume, Vulcano, Aeolian Islands, Italy. Can.Mineral. 48, 1465-1468.

Demartin, F., Gramaccioli, C.M. \& CAmpostrini, I. (2011a): Clinometaborite, natural -metaboric acid, from La Fossa crater, Vulcano, Aeolian Islands, Italy. Can.Mineral. 49, 1273-1279.

Demartin, F., Gramaccioli, C.M., Campostrini, I. \& Castellano, C. (2011b): Cossaite, $\left(\mathrm{Mg}_{0.5}, \mathrm{Al}_{6}\left(\mathrm{SO}_{4}\right)_{6}\left(\mathrm{HSO}_{4}\right) \mathrm{F}_{6} \cdot 36 \mathrm{H}_{2} \mathrm{O}\right.$, a new mineral from La Fossa crater, Vulcano, Aeolian Islands, Italy. Mineralogical Magazine, 75, 2847-2855.

Demartin, F, Campostrini, I., Castellano, C. \& Gramaccioli, C.M. (2012): Argesite, $\left(\mathrm{NH}_{4}\right)_{7} \mathrm{Bi}_{3} \mathrm{Cl}_{16}$, a new mineral from La Fossa Crater, Vulcano, 
Aeolian Islands, Italy.A first example of the $\left[\mathrm{Bi}_{2} \mathrm{Cl}_{10}\right]^{4-}$ anion. Am. Mineral. 97, 1446-1451.

FERRARIS, G., GramaCCIOLI, C.M. (1971): Goldichite, krausite e yavapaiite della solfatara di Pozzuoli. Atti Acc.Sc.Torino 105, 481-492

Fulignati, P. \& SBRAnA, A. (1998): Presence of native gold and tellurium in the active high-sulfidation hydrothermal system of the La Fossa volcano (Vulcano, Italy). Journal of Volcanology and Geochemical Research 86, 187-198.

Garavelli, A. LaViano, R. \& VurRo, F. (1997) Sublimate deposition from hydrothermal fluids at the Fossa crater - Vulcano, Italy. European Journal of Mineralogy, 9 , 423-432.

Garavelli, A. \& VurRo, F. (1994): Barberiite, $\mathrm{NH}_{4} \mathrm{BF}_{4}$, a new mineral from Vulcano, Aeolian Islands,Italy. Am. Mineral. 79, 381-384.

Garavelli, A., Grasso, M.F. \& VurRo, F. (1996): Mineral occurrence and depositional processes at Baia di Levante area (Vulcano Island,Italy) Min. et Petr.Acta 39, 251-261.

Garavelli, A., Mozgova, N.N., Orlandi, P., Bonaccorsi, E., Pinto, D., Mo lo, Y. \& BorodaEV, YU.S. (2005): Rare sulfosalts from Vulcano, Aeolian Islands, Italy. VI. Vurroite $\mathrm{Pb}_{20} \mathrm{Sn}_{2}(\mathrm{Bi}, \mathrm{As})_{22} \mathrm{~S}_{54} \mathrm{Cl}_{6}$, a new mineral species. Can. Mineral. 43, 703-711.

Gebhard, G., Medenbach, O. \& Gebert, W. (1988): Magnesioaubertit, $(\mathrm{Mg}, \mathrm{Cu}) \mathrm{Al}\left(\mathrm{SO}_{4}\right)_{2} \mathrm{Cl} \cdot 14 \mathrm{H}_{2} \mathrm{O}$, ein neues Chlorsulfat von Vulcano, Liparische Inseln. Der Aufschluss 390, 2, 97-102.

ItAliano, F. \& Nuccio, P.M. (1996): Isotopic ratios of helium in fumaroles from Vulcano Island.: Result of the 1993-1995 observations. a Vulcanologica 8, 212 214.

Italiano, F., Pecoraino, G. \& Nuccio, P.M. (1998): Steam output from fumaroles of an active volcano: Tectonic and magmatic-hydrothermal controls on the degassing system at Vulcano (Aeolian arc). J.Geophys.Research 103, 29,829; 29,842.

KeLLER H.L. (1976): Notiz zur Kristallstruktur von $\mathrm{APb}_{2} \mathrm{Cl}_{5}$-Verbindungen; Zeitschrift für Naturforschung 31b, 885.

KRÄMER, V. (1979): Structure of the bismuth-chlorine sulfide $\mathrm{Bi}_{4} \mathrm{Cl}_{2} \mathrm{~S}_{5}$. Acta Cryst. B35, 135-140.

Mazor, E., Cioni, R., Corazza, F., Fratta, M., Magro, G., Matsuo, S., Hirabayashi, J., Shinohara, H., Martini, M., Piccardi, G., Cellini-Legittimo, P. (1988): Evolution of fumarolic gases- boundary conditions set by measured parameters: case study at Vulcano, Italy. Bull. Volcanologique, 50, 71-85.

MerLinO, S. \& SARTORI, F. (1970): Santite, a new mineral phase from Larderello, Tuscany. Can. Mineral. 27, 159-165.

Mitolo, D., Pinto, D., Garavelli, A., Bindi, L., \& Vurro, F. (2009): The role of minor substitutions in the crystal structure of natural challacolloite $\mathrm{KPb}_{2} \mathrm{Cl}_{5}$ and hephaistosite $\mathrm{TlPb}_{2} \mathrm{Cl}_{5}$, from Vulcano (Aeolian archipelago, Italy). Min.Petr. 96, 121-128.

Pinto, D., Balić-Žunić, T., Bonaccorsi, E., Borodaev, Y.S., Garavelli, A., Garbarino, C., Makovicky, E., VurRo, F. (2006a): Rare sulfosalts from 
Vulcano, Aeolian Islands, Italy. VII. Cl-bearing galenobismutite. Can. Mineral. 44, 443-457.

Pinto, D., Balić-Žunić, T., Garavelli, A., Garbarino, C., Makovicky, E., Vurro, F. (2006b): First occurrence of close-to-ideal kirkiite at Vulcano (Aeolian Islands, Italy): chemical data and single-crystal X-ray study. Eur. J. Mineral. 18, 393-401.

Roberts, A.C., Venance, K.E., Seward, T.M., Grice, J.D. \& PAar, W.H. (2006): Lafossaite, a new mineral from the La Fossa Crater, Vulcano, Italy. Mineralogical Record 37, 165-168.

SHCHERBAKOva,Y.P., BAZHENOVA, L.F. \& CHESNOKOV, B.V. (1988): Godovikovite $\mathrm{NH}_{4}(\mathrm{Al}, \mathrm{Fe})\left(\mathrm{SO}_{4}\right)_{2}$, a new ammonium-beating sulfate. Zap. Vses. Mineral. Obsh. 117, 208-211 (in russo).

Skarstad, P.M., Hubbard, C.R., Roth, R.S. \& PARKer, H.S. (1979): The crystal structure of the cation-disordered phase $\left(\mathrm{Tl}_{0.75} \mathrm{~Pb}_{0.25}\right)_{4} \mathrm{Cl}_{5}$. J.Solid State Chemistry 30, 65-78.

Vergasova, L.P., Filatov, S.K., Starova, G.L., Matusevich, G.L. \& Filasova, T.M. (1995): Vlodavetsite, $\mathrm{AlCa}_{2}\left(\mathrm{SO}_{4}\right)_{2} \mathrm{~F}_{2} \mathrm{Cl} \cdot 4 \mathrm{H}_{2} \mathrm{O}$ - a new mineral from volcanic exhalations. Doklady Akad. Nauk. SSSR, 343, 358-360 (in russo).

Vurro, F., Garavelli, A., Garbarino, C., MoËlo, Y. \& Borodaev, Y.S. (1999): Rare sulfosalts from Vulcano, Aeolian Islands, Italy. II. Mozgovaite, $\mathrm{PbBi}_{4}(\mathrm{~S}, \mathrm{Se})_{7}$, a new mineral species. Can. Mineral. 37, 1499-1506.

Zambonini, F., De Fiore, O. \& CAROBbi, G. (1924): Su un solfobismutito di piombo di Vulcano (Isole Eolie). Ann. R. Osservatorio Vesuviano, serie III, 1, 31-36.

ZAmbONINI, F. (1926). Sulla presenza, tra i prodotti dell'attuale attività del Vesuvio, di una varietà cesifera del fluoborato di potassio. Atti R. Accad. Lincei, ser.VI, Classe Sc.fis.mat.e naturali 3, 644-649.

ZAMBOnini, F. \& CAROBBI, G. (1926): Sulla presenza del fluo-silicato di sodio e di quello di potassio fra i prodotti dell'attuale attività del Vesuvio. Rendic. Accad. Lincei, serie 6, 4, 171-175.

ZELENSKI, N. \& BORTNIKOVA, S. (2005): Sublimate speciation at Mutnovsky volcano, Kamchatka. Eur. J. Mineral. 17, 107-118.

Zelenski, M., Balić-Žunić, T., Bindi, L., Garavelli, A., Makovicky, E.R., Pinto, F., VURRO, F. (2006): First occurrence of iodine in natural sulfosalts: the case of mutnovskite $\mathrm{Pb}_{2} \mathrm{AsS}_{3}(\mathrm{I}, \mathrm{Cl}, \mathrm{Br})$, a new mineral from Mutnovsky volcano, Kamchatka Peninsula, Russian Federation. American Mineral. 91, 21-28. 